Table 1. Cumulative Days of Antipseudomonal $\beta$-Lactam Antibiotic Exposure and New Resistance Development

\begin{tabular}{lccc}
$\begin{array}{l}\text { Cumulative Days of } \\
\begin{array}{l}\text { Antipseudomonal } \\
\text { Exposure }\end{array}\end{array}$ & $\begin{array}{c}\text { No. of } \\
\text { Patients }\end{array}$ & $\begin{array}{c}\text { New Resistance } \\
\text { Events, No. (\%) }\end{array}$ & $\begin{array}{c}\text { Hazard Ratio (95\% } \\
\text { Confidence Interval) }\end{array}$ \\
\hline $1-3$ & 1,816 & $38(2.09)$ & 1.00 (reference) \\
\hline $4-6$ & 1,632 & $85(5.21)$ & $1.01(0.93-1.10)$ \\
\hline $7-9$ & 1,249 & $98(7.85)$ & $1.85(1.69-2.02)$ \\
\hline $10-12$ & 709 & $66(9.31)$ & $2.93(2.66-3.24)$ \\
\hline $13-15$ & 474 & $44(9.28)$ & $3.94(3.54-4.39)$ \\
\hline $16-18$ & 326 & $30(9.20)$ & $6.29(5.62-7.04)$ \\
\hline $19-21$ & 234 & $27(11.5)$ & $7.05(6.19-8.02)$ \\
\hline$\geq 22$ & 678 & $56(8.3)$ & $8.52(7.62-9.53)$ \\
\hline
\end{tabular}

\section{Discussion}

Our retrospective cohort study showed the associated rise in the risk of new resistance emergence with increasing duration of antipseudomonal $\beta$-lactam antibiotic exposure in the critically ill does not appear to exhibit a "ceiling effect" as the cumulative duration of exposure increases. This finding is important because it suggests that the risk of new resistance will continue to increase as the duration of exposure increases, regardless of how long the patient has been on antimicrobial therapy.

Recent estimates showing antibiotic resistances accounting for $>2.8$ million infections and $>35,000$ death per year in the United States highlight the need to understand and prevent resistance development. ${ }^{2}$ Minimizing durations of antimicrobial therapy is becoming a pillar of antimicrobial stewardship; however, studies evaluating optimal durations are lacking, and many guideline recommendations for duration of therapy continue to rely on expert opinion which may result in longer than necessary exposures. ${ }^{5-7}$ Our study further highlights the need for further studies evaluating optimal durations for various types of infections as well as studies regarding strategies to limit antimicrobial exposure to the shortest effective duration.

\section{Acknowledgments. None.}

Financial support. Marin Kollef s efforts were supported by the Barnes-Jewish Hospital Foundation.

Conflicts of interest. All authors report no conflicts of interest relevant to this article.

\section{References}

1. Marston HD, Dixon DM, Knisely JM, Palmore TN, Fauci AS. Antimicrobial resistance. JAMA 2016;316:1193-1204.

2. Antibiotic resistance threats in the United States, 2019. Centers for Disease Control and Prevention website. https://www.cdc.gov/drugresistance/pdf/ threats-report/2019-ar-threats-report-508.pdf. Published 2019. Accessed December 10, 2019.

3. Rhodes A, Evans LE, Alhazzani W, et al. Surviving Sepsis Campaign: international guidelines for management of sepsis and septic shock: 2016. Crit Care Med 2017;45:486-552.

4. Teshome BF, Vouri SM, Hampton N, Kollef MH, Micek ST. Duration of exposure to antipseudomonal beta-lactam antibiotics in the critically ill and development of new resistance. Pharmacotherapy 2019;39:261-270.

5. Barlam TF, Cosgrove SE, Abbo LM, et al. Implementing an antibiotic stewardship program: guidelines by the Infectious Diseases Society of America and the Society for Healthcare Epidemiology of America. Clin Infect Dis 2016;62(10):e51-e77.

6. Hayashi Y, Paterson DL. Strategies for reduction in duration of antibiotic use in hospitalized patients. Clin Infect Dis 2011;52:1232-1240.

7. Cole KA, Rivard KR, Dumkow LE. Antimicrobial stewardship interventions to combat antibiotic resistance: an update on targeted strategies. Curr Infect Dis Rep 2019;21(10):33.

\title{
Silent clonal spread of vancomycin-resistant Enterococcus faecalis ST6 and ST525 colonizing patients at hospital admission in Natal, Brazil
}

\author{
Emília S. de Oliveira MS ${ }^{1, a}$, Ana R. Freitas PhD²,a, Luísa Peixe $\mathrm{PhD}^{2}$, Carla Novais $\mathrm{PhD}^{2}$ (i) and M. Celeste Melo $\mathrm{PhD}^{1}$ \\ ${ }^{1}$ Laboratory of Medical Bacteriology, Department of Microbiology and Parasitology, Federal University of Rio Grande do Norte, Natal, Rio Grande do Norte, Brazil
} and ${ }^{2}$ UCIBIO/REQUIMTE Applied Molecular Biosciences Unit, Department of Biological Sciences, Faculty of Pharmacy, University of Porto, Porto, Portugal

To the Editor-Infections and gut colonization with vancomycinresistant enterococci (VRE) have been increasingly reported in hospitalized patients from different regions of Brazil, where difficulties in controlling VRE colonization have been noted. ${ }^{1-4}$ Patient

\footnotetext{
Author for correspondence: Carla Novais, E-mail: casilva@ff.up.pt

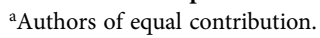

Cite this article: de Oliveira ES, et al. (2020). Silent clonal spread of vancomycinresistant Enterococcus faecalis ST6 and ST525 colonizing patients at hospital admission in Natal, Brazil. Infection Control \& Hospital Epidemiology, 41: 485-487, https:// doi.org/10.1017/ice.2020.24
}

colonization with VRE is a major risk factor for developing subsequent infections with these strains. ${ }^{1,5}$ The first VRE description dates from 2011 (M. Celeste Melo, personnel data), and VRE infections among hospitalized patients from Natal city (northeastern Brazil) remain low, contrasting with the high rates of VRE infection and colonization of the patients in the southern and southeastern regions, where they have occurred since $1998 .^{1-3}$ For early recognition of silent interhospital VRE transmission through colonized patients as in other parts of Brazil, we aimed to search and characterize VRE colonization strains from patients known to have a previously history of hospitalization. 
Table 1. Genetic Characterization of 18 Representative Enterococcus faecalis With Different Epidemiological Backgrounds

\begin{tabular}{|c|c|c|c|c|c|c|c|c|c|c|c|c|c|c|c|c|c|c|}
\hline \multirow[b]{2}{*}{ PFGE } & \multirow[b]{2}{*}{ MLST } & \multirow[b]{2}{*}{ Hospital } & \multirow[b]{2}{*}{ Patient $^{a}$} & \multirow[b]{2}{*}{ Sample } & \multirow[b]{2}{*}{ Date } & \multicolumn{6}{|c|}{ Acquired Antibiotic Resistance Genes } & \multicolumn{7}{|c|}{ Virulence-Associated Genes } \\
\hline & & & & & & vanA & $\operatorname{erm}(B)$ & $\operatorname{tet}(M)$ & $a c\left(6^{\prime}\right)-l e-a p h\left(2^{\prime \prime}\right)-I a$ & $\operatorname{aph}\left(3^{\prime}\right)-I I I$ & ant(6)-Ia & gelE-fsrB & cyl & ace & agg & efaAfs & $e b p A B C$ & srtA \\
\hline A & ST525 & $\mathrm{HB}$ & 17 & Perianal swab & 13.09 .2015 & + & + & + & + & - & - & + & + & + & - & + & + & + \\
\hline A & ST525 & HA & 22 & Perianal swab & 11.10 .2015 & + & + & + & + & - & - & + & + & + & - & + & + & + \\
\hline A & ST525 & $\mathrm{HA}$ & 18 & Catheter tip & 20.10 .2015 & + & + & + & + & - & - & + & + & + & - & + & + & + \\
\hline A & ST525 & Home (OP) & 28 & Perianal swab & 08.01 .2016 & + & + & + & - & - & - & + & + & + & - & + & + & + \\
\hline A & ST525 & $\mathrm{HF}$ & 36 & Perianal swab & 09.03 .2016 & + & + & + & + & - & - & + & + & + & - & + & + & + \\
\hline A & ST525 & Home (OP) & 46 & Ulcer secretion & 29.11 .2016 & + & + & + & + & - & - & + & + & + & - & + & + & + \\
\hline A & ST6 & $\mathrm{HC}$ & 3 & Urine & 01.06 .2015 & + & + & + & + & - & - & + & + & + & + & + & + & + \\
\hline A & ST6 & $\mathrm{HA}$ & 11 & Perianal swab & 30.06 .2015 & + & + & + & + & + & + & + & + & + & + & + & + & + \\
\hline B & ST525 & $\mathrm{HE}$ & 2 & Perianal swab & 18.05 .2015 & + & + & + & + & - & - & + & + & + & + & + & + & + \\
\hline B & ST525 & $\mathrm{HF}$ & 10 & Ocular secretion & 26.06.2015 & + & + & + & + & - & - & + & + & + & + & + & + & + \\
\hline B & ST525 & $\mathrm{HC}$ & 38 & Perianal swab & 12.03 .2016 & + & + & + & + & - & - & + & + & + & - & + & + & + \\
\hline B & ST525 & $\mathrm{HB}$ & 41 & Urine & 08.04 .2016 & + & + & + & + & - & - & + & + & + & - & + & + & + \\
\hline B & ST525 & $\mathrm{HB}$ & 17 & Perianal swab & 30.11 .2016 & + & - & + & + & - & - & + & + & + & - & + & + & + \\
\hline B & ST525 & $\mathrm{HC}$ & 47 & Perianal swab & 12.12 .2016 & + & + & + & + & - & - & + & + & + & + & + & + & + \\
\hline B & ST525 & $\mathrm{HE}$ & 26 & Perianal swab & unknown & + & + & + & + & - & - & + & + & + & + & + & + & + \\
\hline B & ST6 & $\mathrm{HB}$ & 9 & Bone fragment & 25.06 .2015 & + & + & + & + & + & + & + & + & + & + & + & + & + \\
\hline B & ST6 & $\mathrm{HC}$ & 9 & Perianal swab & 02.07 .2015 & + & + & + & + & + & + & + & + & + & + & + & + & + \\
\hline C & ST6 & HA & 45 & Perianal swab & unknown & + & + & + & + & + & + & + & + & + & + & + & + & + \\
\hline
\end{tabular}

Note. PFGE, pulsed-field-gel-electrophoresis; MLST, multilocus sequence typing; OP, outpatient

aWhen the same patient carried VRE in $>1$ occasion, the data are shown in bold.

\begin{tabular}{|l|l|}
\hline Glycopetides & Aminoglycosides \\
\hline Macrolides & Secreted factor for tissue damage \\
\hline Tetracyclines & Adhesins and biofilm-associated genes \\
\hline
\end{tabular}


From January 2015 to December 2016, 55 VRE isolates were recovered (chromID-VRE) from perianal swabs of 44 inpatients at 5 hospitals (range, 36-257 beds) in Natal (public hospitals A, $\mathrm{B}$, and F; public-private hospital C, or private hospital E) during hospital admission and of 3 outpatients when receiving domiciliary nursing care at their own home. Most of these patients had previous hospitalization episodes. Six additional VRE were collected from different clinical specimens during the hospitalizations (in hospitals $\mathrm{A}, \mathrm{B}, \mathrm{C}, \mathrm{D}$, and $\mathrm{F}$ ) of 6 patients ( 2 in common with those from the cohort of 44 patients). Species identification and search of antibiotic resistance, virulence, and plasmid replicase genes were conducted using polymerase chain reaction testing (PCR) in isolates representative of different clones and epidemiological contexts. ${ }^{6,7}$ Antibiotic susceptibility was assessed using disk diffusion (ie, for ampicillin, tetracycline, chloramphenicol, gentamicin, erythromycin, and streptomycin), Etest (vancomycin), agar dilution (teicoplanin), or broth microdilution (linezolid) following the European Committee on Antimicrobial Susceptibility Testing (EUCAST) clinical breakpoints (http://www.eucast.org/clinical_breakpoints/) or, when not possible, Clinical and Laboratory Standards Institute (CLSI) breakpoints. Clonal relationships were firstly evaluated by SmaI pulsed-field gel electrophoresis (PFGE) in the 61 VRE and by multilocus sequence typing (MLST) in 18 Enterococcus faecalis $(E f s)$ (ie, different PFGE types, patients, and hospitals). ${ }^{6}$

The VRE were identified as Efs $(\mathrm{n}=60)$ and E. faecium $(\mathrm{n}=1)$, all carrying vanA. In this study, we only proceeded with the $E f$; all were multidrug resistant $(\mathrm{MDR})$ and exhibited resistance to vancomycin (range, 16 to $>256 \mathrm{mg} / \mathrm{L}$ ), teicoplanin (range, 4-64 $\mathrm{mg} / \mathrm{L}$ ), ciprofloxacin, tetracycline, and erythromycin. Most were also resistant to gentamicin (93\%) and streptomycin (42\%). PFGE established 3 pulsotypes among the $60 \mathrm{VREfs}$ : 30 for pulsotype A, 29 for pulsotype $\mathrm{B}$, and 1 for pulsotype $\mathrm{C}$. The dominant pulsotypes $A$ and $B$ were dispersed among all institutions and were detected throughout the study period. Representative VREfs from pulsotypes $A$ and $B$ were identified as sequence type 6 (ST6; $n=4: 2$ of pulsotype A and 2 of pulsotype B) or ST525 ( $n=13: 6$ of pulsotype A and 7 of pulsotype B). Pulsotype-C was also an ST6 (Table 1). Examples of $E f s$ strains showing the same or similar pulsotypes but clustering in different sequence types have previously been described. ${ }^{8}$ ST6 is dispersed worldwide mostly in association with nosocomial infections, whereas ST525 has only been documented in Brazil southeastern regions (VREfs linezolid-resistant infections; https:// pubmlst.org), ${ }^{4,9}$ suggesting the dispersion of relevant strains of such lineage in this country. A patient was colonized with both ST525 A and B clones collected 14 months apart. The same PFGE-B/ST6 Efs was associated with 2 isolates from the same patient (bone fragment and colonization) obtained 7 days apart in different hospitals (Table 1). Moreover, vanA plasmid replicases from the $18 \mathrm{VREfs}$ were identical ( $\mathrm{rep}_{9}$ from pheromone-responsive pTEF2/pAD1 plasmids) and known to transfer highly efficiently, which could have enhanced vancomycin-resistance spread also by horizontal gene transfer. Among the $18 \mathrm{VREfs}$, all carried $\operatorname{vanA} \operatorname{erm}(B), \operatorname{tet}(M)$, $a a c\left(6^{\prime}\right)$-aph(2"), whereas $a p h\left(3^{\prime}\right)-I I I$, and ant(6)-Ia were variable. Putative virulence factors included adhesins, biofilm-associated genes, and survival genes (eg, ace, gelE, and $a g g / c y l$ ) previously associated with dominant lineages of human infections (Table 1). ${ }^{10}$

In summary, patients with a previous history of hospitalization and who had been admitted to different hospitals of Natal were colonized with 2 VREfs clonal lineages with potential to cause infection, one closely related to global ST6-VREfs and the other more region specific (ST525). ${ }^{4,9}$ The detection of several $E f s$ strains with the same PFGE type associated with different sequence types (via MLST) highlights both the limited accuracy of these methods to identify identical $E f s$ strains and the need to use methods with a higher resolution to better follow transmission events between patients and institutions. The identification of 2 specific VREfs clones is reminiscent of the emergence of VRE in other regions of Brazil in previous years, where VREfs strains are now being replaced by dominant vancomycin-resistant hospital-associated E. faecium. ${ }^{2,3}$ Our results highlight the need for better hygiene measures, systematic colonization survey of transferred patients, patient isolation, and antimicrobial stewardship to prevent future epidemic and endemic scenarios associated with infection in hospitals from Natal. Of special concern is the transfer of such VRE to the community level by colonized patients with domiciliary nursing, which represents another potential level of transmission requiring additional strategies to control their spread.

\section{Acknowledgments. None.}

Financial support. This work was supported by the Coordenação de Aperfeiçoamento de Pessoal de Nível Superior (CAPES) and by funding from the Applied Molecular Biosciences Unit - UCIBIO which is financed by national funds from FCT (UIDB/04378/2020). A.R.F. gratefully acknowledges the junior research position funded by FCT/MCTES through national funds (grant no. CEECIND/02268/2017, Individual Call to Scientific Employment Stimulus 2017).

Conflicts of interest. All authors report no conflicts of interest relevant to this article.

\section{References}

1. Campos PA, Batistão DWF, Gontijo-Filho PP et al. A sustained endemic outbreak of vancomycin-resistant Enterococcus faecium: a 30-month surveillance study. Scand J Infect Dis 2014; 46:547-554.

2. Resende M, Caierão J, Prates JG, et al. Emergence of vanA vancomycinresistant Enterococcus faecium in a hospital in Porto Alegre, South Brazil. J Infect Dev Ctries 2014;8:160-167.

3. Sacramento AG, Zanella RC, Esposito F, et al. Changed epidemiology during intra- and interhospital spread of high-risk clones of vanAcontaining Enterococcus in Brazilian hospitals. Diagn Microbiol Infect Dis 2017;88:348-351.

4. de Almeida LM, de Araujo MRE, Iwasaki MF et al. Linezolid resistance in vancomycin-resistant Enterococcus faecalis and E. faecium isolates in a Brazilian hospital. Antimicrob Agents Chemother 2014;58:2993-2994.

5. Alevizakos M, Gaitanidis A, Nasioudis D, et al. Colonization with vancomycin-resistant enterococci and risk for bloodstream infection among patients with malignancy: a systematic review and meta-analysis. Open Forum Infect Dis 2016;4(1):ofw246.

6. Freitas AR, Novais C, Duarte B, et al. High rates of colonisation by ampicillin-resistant enterococci in residents of long-term care facilities in Porto, Portugal. Int J Antimicrob Agents 2018;51:503-507.

7. Freitas AR, Novais C, Tedim AP, et al. Microevolutionary events involving narrow host plasmids influences local fixation of vancomycin resistance in Enterococcus populations. PLoS One 2013;8:e60589.

8. Freitas AR, Coque TM, Novais C, et al. Human and swine hosts share vancomycin-resistant Enterococcus faecium CC17 and CC5 and E. faecalis CC2 clonal clusters harboring Tn1546 on indistinguishable plasmids. J Clin Microbiol 2011;49:925-31.

9. Santos BA, Oliveira JS, Cardoso NT, et al. Major globally disseminated clonal complexes of antimicrobial-resistant enterococci associated with infections in cancer patients in Brazil. Infect Genet Evol 2017;55: 56-62.

10. Raven KE, Reuter S, Gouliouris T, et al. Genome-based characterization of hospital-adapted Enterococcus faecalis lineages. Nat Microbiol 2016;1:15033. 